\title{
HIGH POWER COAX WINDOW
}

\author{
M. Neubauer, R. Sah, A. Dudas, Muons, Inc., Batavia, IL, 60510, U.S.A \\ B. Rimmer, T. Elliot, M. Stirbet, JLab, Newport News, VA USA.
}

\section{Abstract}

A superconducting RF (SRF) power coupler capable of handling $500 \mathrm{~kW} \mathrm{CW} \mathrm{RF} \mathrm{power} \mathrm{is} \mathrm{required} \mathrm{for} \mathrm{present}$ and future storage rings and linacs. There are over 35 coupler designs for SRF cavities ranging in frequency from 325 to $1500 \mathrm{MHz}$. Coupler windows vary from cylinders to cones to disks and RF power couplers will always be limited by the ability of ceramic windows and their matching systems to withstand the stresses due to non-uniform heating from dielectric and wall losses, multipactor, and mechanical flexure. We propose a novel robust co-axial SRF coupler design which uses two windows manufactured with compression ring technology and no matching elements in the coax line. This technology will allow the use of high thermally conductive materials for cryogenic windows because the braze joint will be in compression. In Phase II a scaled down version of the $500 \mathrm{~kW} \mathrm{CW}$ double window assembly will be built and tested at high power in order to finalize the design for the full size. The processes used to build compressed coax windows is scalable to larger diameter coax and to higher power levels.

\section{INTRODUCTION AND TECHNICAL APPROACH}

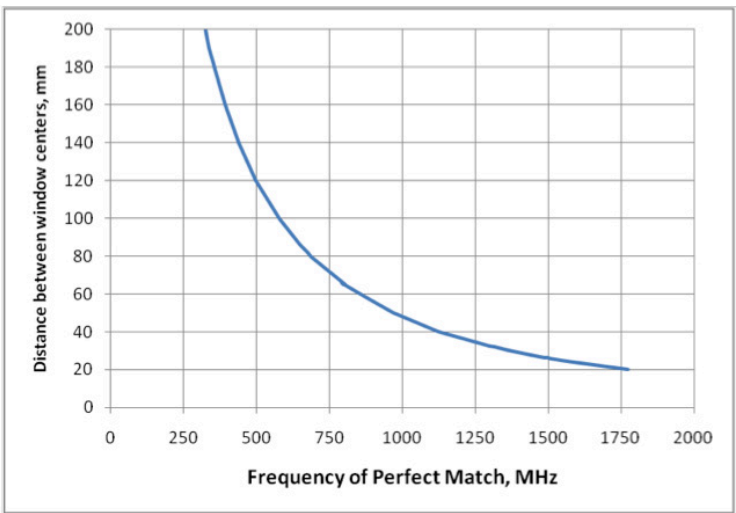

Figure 1: Two alumina windows $7.6 \mathrm{~mm}$ thick modeled in EIA 3-1/8 coax for the distance between them which creates a near perfect match

When two coax windows are placed in a length of line, the distance between them can be adjusted to create a perfect match. That distance is approximately a quarterwavelength. The thickness of the window and its dielectric constant determines the exact distance for the cancellation of reflections. For EIA 3-1/8 $50 \mathrm{ohm}$ coax line, the ceramic window has the same dimensions as the coax (76.9 $\mathrm{mm}$ and $33.4 \mathrm{~mm})$. In our Phase I construction of a compressed window we used a window thickness of $7.6 \mathrm{~mm}$. Using those dimensions the optimum spacing for various frequencies can be determined by various finite element programs. For EIA 3-1/8 coaxial line, Figure 1 shows the window spacing as a function of frequency, which provides a near perfect match.

Calculation of power dissipation in the windows with $100 \mathrm{~kW}$ of input power is shown in Table I. This is not the whole story for power dissipation. What is not included is the loss from the metalizing that is on both the ID and the OD of the coax window. We know from previous work on coaxial windows that this number is about equal to the dielectric losses.

Table I: Comsol Calculations of power dissipated and the maximum gradient in the EIA 3-1/8 coax line with alumina windows (at room temperature) with $\tan \delta=.0001$ at $100 \mathrm{~kW}$ input power.

\begin{tabular}{|c|c|c|}
\hline $\begin{array}{c}\text { Freq } \\
(\mathrm{MHz})\end{array}$ & $\begin{array}{c}\text { Watts ina } \\
\text { windowat } \\
100 \mathrm{~kW} \text { Pin }\end{array}$ & $\begin{array}{c}\text { Gradient at } \\
100 \mathrm{~kW} \\
(\mathrm{~V} / \mathrm{m})\end{array}$ \\
\hline 1300 & 18.6 & $2.29 \mathrm{E}+05$ \\
\hline 805 & 12.2 & $2.56 \mathrm{E}+05$ \\
\hline 650 & 10.7 & $2.72 \mathrm{E}+05$ \\
\hline 325 & 5.14 & $3.77 \mathrm{E}+05$ \\
\hline
\end{tabular}

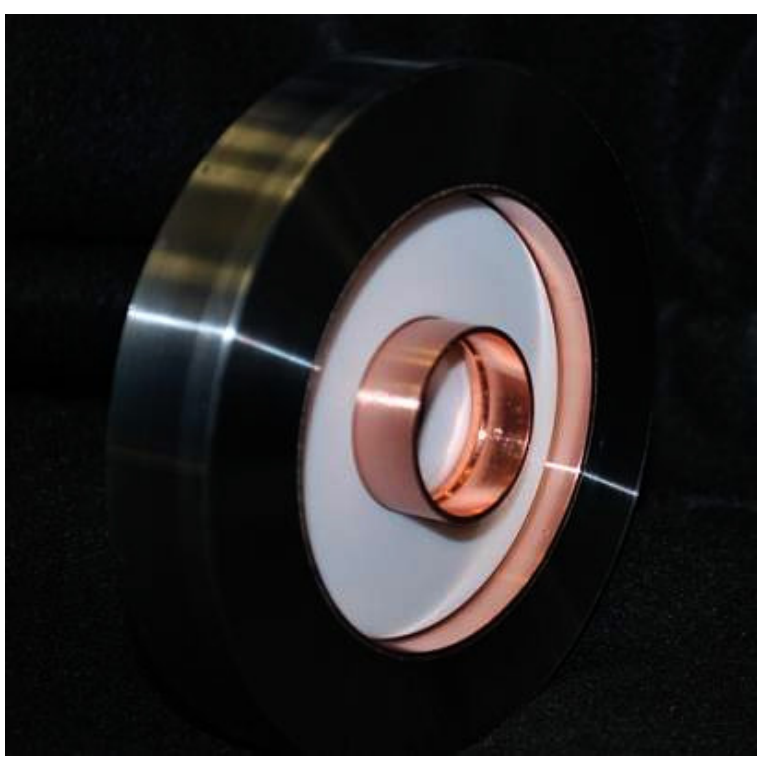

Figure 2: Completed brazed window in a compression ring

The inner conductor sub-assembly braze was the first crucial element in this design. The problem that needed to be addressed was the undesirable stresses that would occur on the braze joint between the coax window and the inner conductor during cool down from brazing. To

07 Accelerator Technology 
accomplish this, a sub-assembly was brazed as shown in Figure 3. This worked quite well without too much deformation of the copper inner conductor. A machine step was included if necessary to true up the OD of this sub assembly if the deformation from the braze cycle created a problem. As it turned out, the copper was extruded and the ends collapsed slightly, which was not an issue.

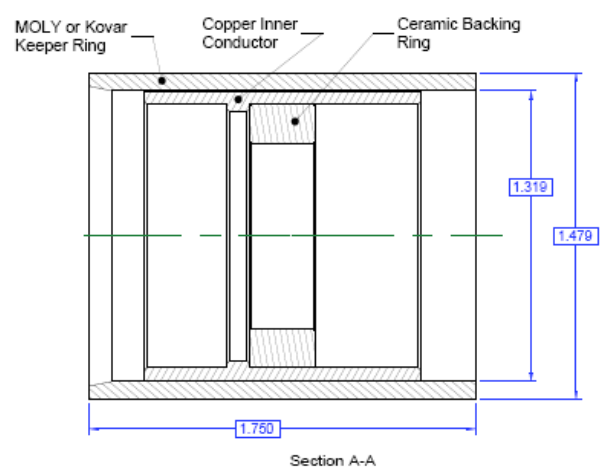

Figure 3: Center Conductor sub-assembly braze.

Shown below in Figure 4 is the detail in the subassembly braze for the critically important buffer layer on the ID of the stainless steel compression ring. It was decided to braze a copper plug into the stainless steel and post machine the ID of the copper centered to the OD of the stainless steel ring. This process was perfected by JLAB in a prior construction of compressed waveguide windows. This is an improvement over previously plated buffer layers in other compression window designs.
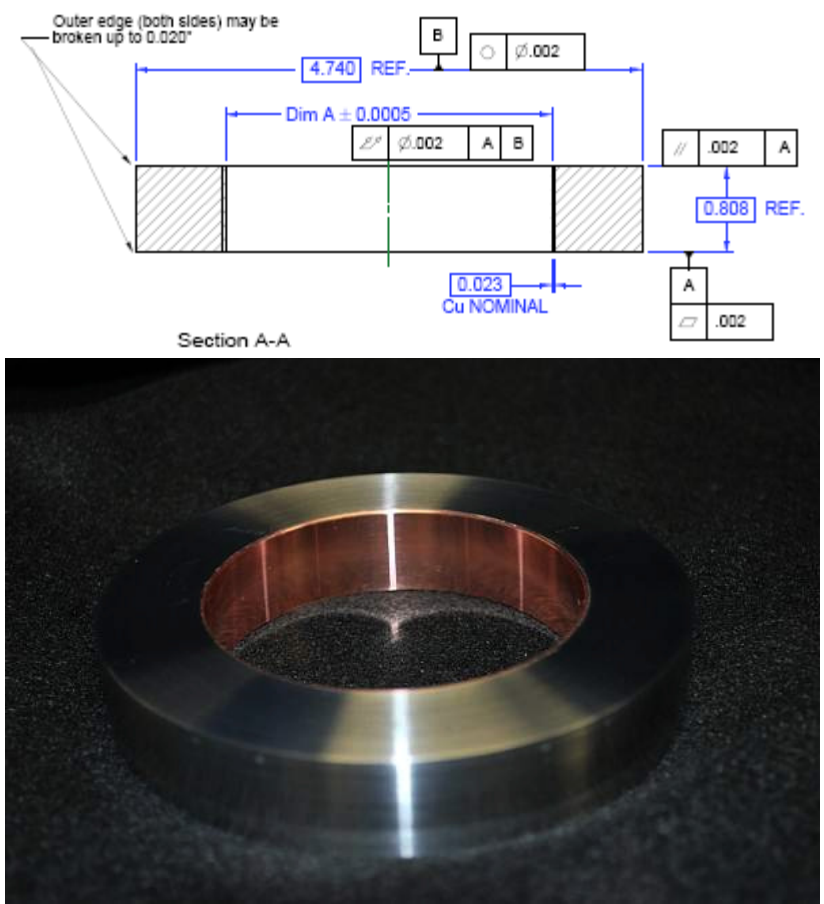

Figure 4: Compression Ring post machining

The final assembly braze cycle has not changed from the original work done earlier [1]. The object is to make the braze inside a large moly keeper ring that confines the stainless steel compression ring at braze temperatures. The setup in the brazing furnace is shown in Figure 5.

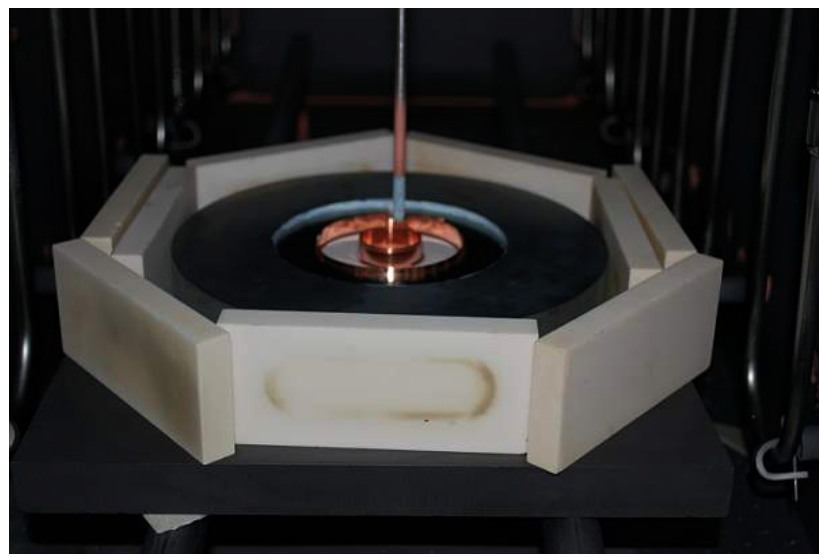

Figure 5 All the sub assemblies and moly keeper ring position in the braze furnace. The ceramic blocks are used to buffer the heat from the elements of the furnace and assure uniform heating of the parts.

During cool down, the compression ring shrinks down around the window and compresses it. This is shown in Figure 6. Note that the ID of the compression ring is about .020 smaller than what it was at the beginning of the process.

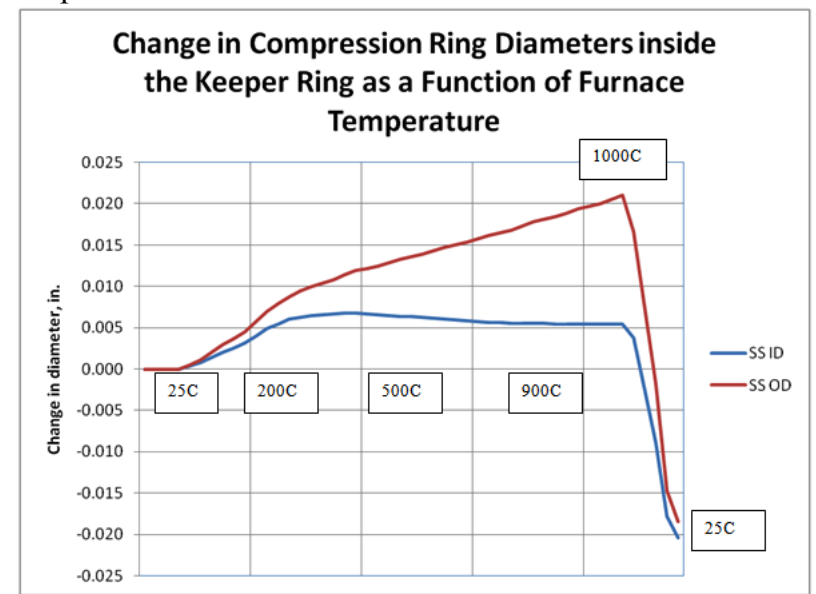

Figure 6: ANSYS calculations of the diameters of the compression ring during the braze cycle.

Another critical element of the brazing process is the stress relieving done at $440^{\circ} \mathrm{C}$ during the cool down process. This critical step resulted from a number of experiments by the PI showing that the copper buffer layer may have built in sheer stresses, resulting from the differentials between the shrinkage of the copper, the stainless steel, and the ceramic in the axial direction [1]. These stresses are the most damaging to the edge of the ceramic, often resulting in edge cracks if not properly stress relieved

\section{Accelerator Technology}




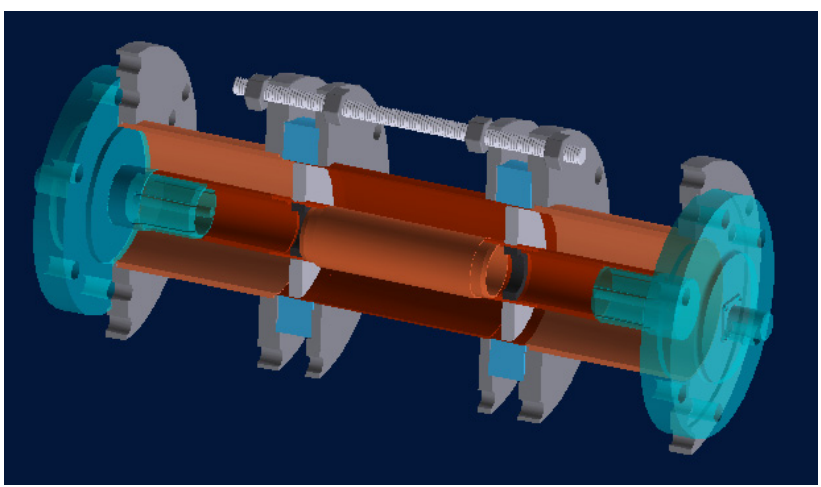

Figure 7: Double window cold test fixture.

\section{FUTURE WORK}

In the Phase II work window materials will be used in the assembly processes ranging from soft boron nitride to lossy aluminum nitride and sapphire. A scaled down version of the ultimate double window design for $500 \mathrm{~kW}$ $\mathrm{CW}$ at $750 \mathrm{MHz}$ will be built and stress tested at cryogenic temperatures as well as room temperature with varying pressures and gases. As a result of these comprehensive tests, a design scaled up to full power $\mathrm{CW}$ can be reliably produced.

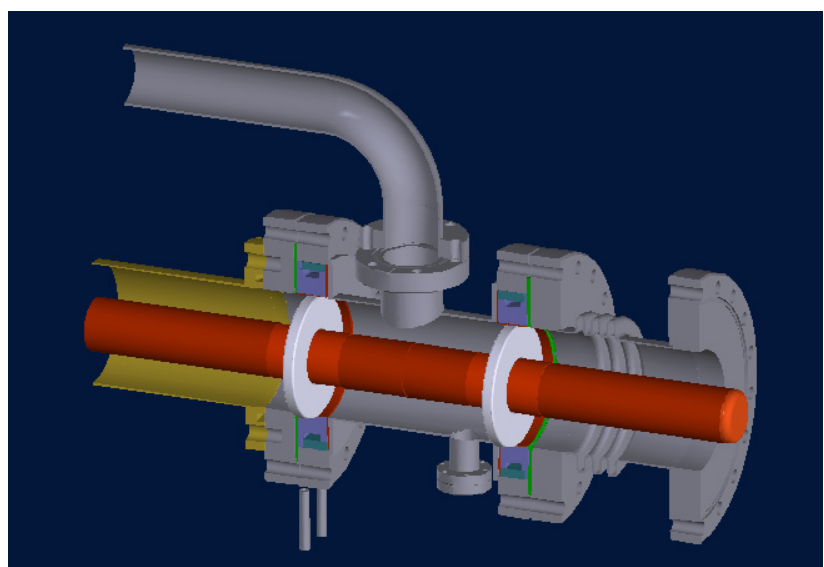

Figure 8: Conceptual drawing of the double window system

The double window concept has many advantages of other RF coupler designs. Primarily there are no impedance matching elements, no changes in diameter of the ID or the OD of the coax line. This will have significant advantages for processing times as the multipactor bands are run through. In addition, the gap between the windows can be filled with a gas such as SF6, or N2, to improve power handling capability. Or simply as a vacuum barrier to the outside environment, should the cold window experience any problems.

The tuning mechanisms have to be designed, but one option is to change the spacing between the windows. The other is the standard means using bellows as shown in Figure 8.

\section{CONCLUSIONS}

We have presented a means for making double windows with compression rings to increase the operating temperature and remove the tensile stresses that tend to break them. The design process, fixturing and tooling can be scaled to larger diameter coax lines. The $500 \mathrm{~kW} \mathrm{CW}$ application at $750 \mathrm{MHz}$ can use this window technology once we have worked out the design limits at the scaled down version. This will allow us to understand what diameter would best fit the high power application.

\section{REFERENCES}

[1] Neubauer, M., et. al. "High-power RF window design for the PEP-II B-factory." SLAC-PUB-6553, EPAC94 